\section{Comparison of efficiency and output power of optimal Er-doped superfluorescent fibre sources in different configurations}

\author{
L.A. Wang and C.D. Chen
}

Indexing terms: Fibre lasers, Fhorescence

The efficiencies and output power of Er-doped superfluorescent fibre sources of three major configurations are compared when pumped near $980 \mathrm{~nm}$ and operated in their optimal either pumppower independent, or insensitive mean wavelength regions for the first time. It is shown the double-pass backward source has a better performance than the others.

Introduction: Er-doped superfiuorescent fibre sources (SFSs) pumped near $980 \mathrm{~nm}$ in a singlepass backward (SPB) configuration have recently been extensively studied for navigation-grade fibre optical gyroscopes (FOGs) [1 - 4]. Other possible SFS configurations are double pass-backward (DPB), singlepass forward (SPF) and double-pass forward (DPF), etc. Since the mean wavelength stability of an SFS determines the detection accuracy of an FOG, it is very important to minimise the temperature dependence of the mean wavelength [1]. Among the temperature-induced effects which cause mean wavelength variation, the pump-power dependent effect, i.e. $\partial \bar{\lambda}_{\text {soured }} \partial P_{\text {pumpro }}$, varies with configuration, and should be either reduced or, if possible, completely eliminated. So far, only Er-doped SFSs in SPB [2, 4] and DPB [5] configurations have been demonstrated to have pump-power independent mean wavelength operations, i.e. $\partial \bar{\lambda}_{\text {sourcec }} / \partial P_{\text {pump }}=0$.

In addition to the requirement of mean wavelength stability, high output power and pump efficiencies are desirable for a broadband SFS. From the system viewpoint, high output power can help increase the total length of fibre in the loop, and thus relax the sensitivity requirement in a measurement system; whereas high pump efficiency implies that high SFS output power may be obtained using a compact laser diode rather than by a solid state laser such as Ti-sapphire, which would facilitate the practical implementation of a FOG.

The maximum slope efficiency of an Er-doped SPB SFS pumped near $980 \mathrm{~nm}$ was $31 \%$ obtained by utilising either 78 or $23 \mathrm{~m}$ EDFs [4], and the output power was $26 \mathrm{~mW}$ at $100 \mathrm{~mW}$ pump power [2]. As for the forward signals, an SPF SFS is of less interest because of its poor quantum conversion efficiency. In contrast, the simulation results indicated that a DPF SFS could have a reduced efficiency when operating in a pump-power insensitive mean wavelength region [1]. In this Letter, we compare the efficiencies and output power of Er-doped SFSs in three major configurations, i.e. SPB-, DPB-, and DPF-ones, each operating in its optimal mean wavelength region, which is either pump-power independent or pump-power insensitive. The results show that a DPB SFS can have a better performance than the SFSs in other configurations in the following aspects: pump efficiency, output power, and linewidths.

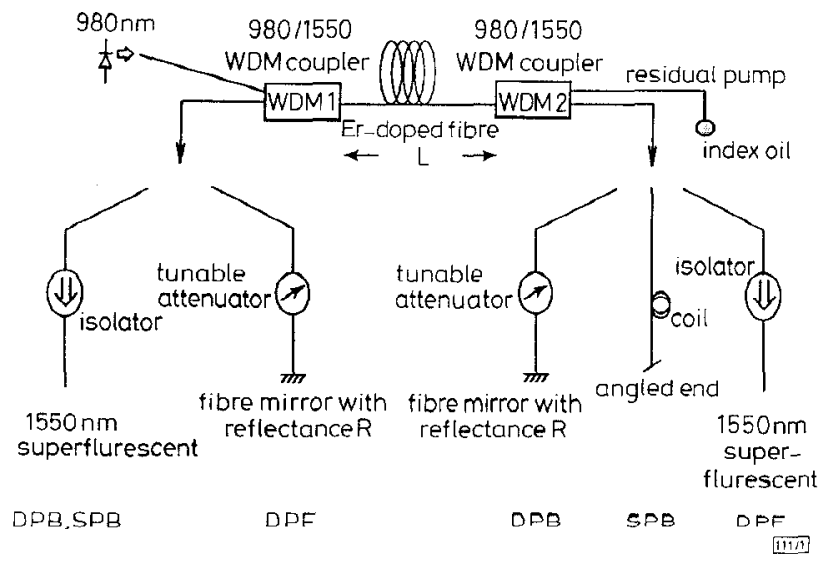

Fig. 1 Experimental arrangement for characterising $D P B, D P F$ and $S P B$ SFSS

Experiment and discussion: The experimental setup used to characterise SPB, DPB and DPF Er-doped SFSs is schematically shown in Fig. 1. Pump power was provided by a laser diode with a maximum output power of $82 \mathrm{~mW}$ measured at the EDF input end. The EDF had $\sim 600 \mathrm{wt}$ ppm doping concentration, and was codoped with $\mathrm{Al}_{2} \mathrm{O}_{3}$ and $\mathrm{GeO}_{2}$. The numerical aperture was 0.16 , and singlemoded when the EDF was pumped near $980 \mathrm{~nm}$. A fibre mirror with controllable reflectance realised by incorporating a fibre mirror $(R \simeq 90 \%)$ and a tunable precision attenuator was spliced to one of the WDMs to reflect the forward and the backward ASE signals in DPB and DPF configurations, respectively. In the SPB configuration, the $1550 \mathrm{~nm}$ signal output end of WDM2 was coiled and angle-cleaved to reduce the optical back reflection. To reduce the optical feedback at the signal output end, a polarisation-insensitive isolator with $-59 \mathrm{~dB}$ isolation was incorporated. The residual pump end of WDM 2 was index matched to prevent back reflection. The mean wavelength [3], linewidth [1] and pumping efficiency were calculated based on the measured spectra.

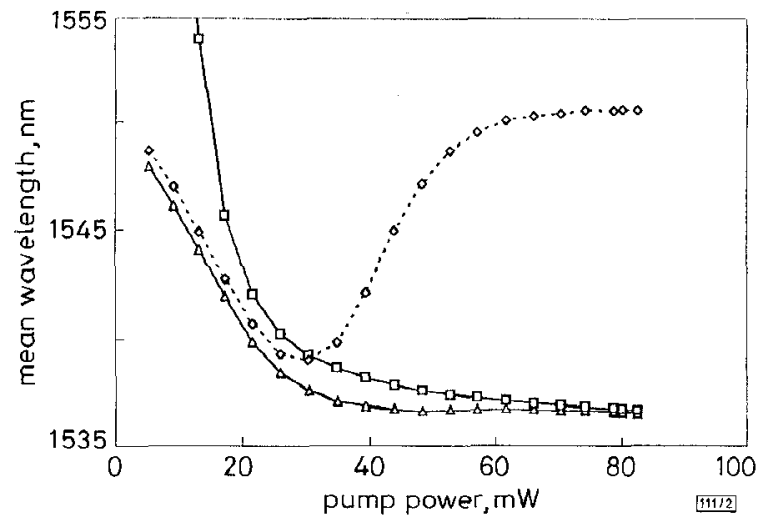

Fig. 2 Mean wavelength variation against pump power for various configurations

$\triangle$ SPB $11 \mathrm{~m}$
$\square$ DPF $6 \mathrm{~m}$
$\diamond \mathrm{DPB} 11 \mathrm{~m}$

The dependence of mean wavelength upon pump power and the corresponding pump power efficiencies were characterised, and the results are shown in Figs. 2 and 3, respectively. For both doublepass configurations, there was no applied attenuation, and the effective fibre mirror reflectance was $\sim 50 \%$ after correction for the insertion losses of the WDM and the attenuator. The EDF lengths were chosen to be 6,11 and $11 \mathrm{~m}$ for DPF, DPB and SPB configurations, respectively. The utilisation of such lengths was based on the following optimal considerations, referring to Fig. 2: (i) for SPB configuration, nearly saturated output power was obtained with a $\partial \bar{\lambda}_{\text {source }} / \partial P_{\text {pump }}$ tradeoff of $\sim-3 \mathrm{ppm} / \mathrm{mW}$ at $80 \mathrm{~mW}$ pump, although the $\partial \bar{\lambda}_{\text {source }} / \partial P_{\text {pump }}=0$ operation occurred at $\sim 50 \mathrm{~mW}$ pump; (ii) for DPB configuration, $\partial \bar{\lambda}_{\text {source }} \partial P_{\text {pump }}=0$ was obtained at $80 \mathrm{~mW}$ pump; (iii) for DPF configuration, the maximum pump efficiency and a relatively smaller $\partial \bar{\lambda}_{\text {soured }} / \partial P_{\text {pump }}$ of $\sim 13 \mathrm{ppm} / \mathrm{mW}$ were obtained, as compared to other lengths. Within the maximum pump power available, however, no pump-power independent mean wavelength operation was found for the SFS in the DPF configuration at various EDF lengths ranging from 5 to $10 \mathrm{~m} \mathrm{[6].}$ As depicted in Fig. 3, for the DPF SFS, the threshold pump power was $\sim 20 \mathrm{~mW}$, while the SFSs in DPB and SPB configurations had similar but higher pump thresholds $(-30 \mathrm{~mW}$ for SPB and $\sim 32 \mathrm{~mW}$ for DPB). The lower threshold of the DPF SFS is attributed to the use of a shorter EDF length. The measured slope efficiency of the DPB SFS was $\sim 52 \%$, higher than those obtained in SPB $(\sim 27 \%)$ and DPF $(\sim 38 \%)$ configurations. The efficiency also represents the highest value obtained from an Er-doped SFS pumped near $980 \mathrm{~nm}$. Such a high efficiency is attributed to the double pass feature and the high pump being injected closer to the signal output end [7]. In addition, as shown in Fig. 3, at higher pump power levels, the output power from a DPB SFS was larger than that from both DPF and SPB SFSs. For example, at the maximum pump power, an output power of 26,14 and $24 \mathrm{~mW}$ was obtained for the DPB, SPB and DPF configurations, respectively. The simulation results indicate that the slopes of the three configurations remain the same as pump power continues to increase [7], therefore, it is expected that the output power will become larger as a pump laser diode with higher power is utilised. 
It is known that an SFS is excess-noise limited in high power operation [8]. The signal-to-noise ratio (SNR) is then determined only by the source linewidth and the detector bandwidth. The corresponding linewidths of the SFSs measured at the optimal conditions mentioned previously were 27,14 and $12 \mathrm{~nm}$ for DPB, SPB and DPF configurations, respectively. The SFS in the DPB configuration therefore showed a better performance not only in pump efficiency and output power but also in linewidth. Additionally, it should be noted that all the results of the double-pass configurations were obtained with an effective mirror reflectance of $\sim 0.5$. The effects of varying mirror reflectance of a DPB SFS was characterised and will be reported elsewhere. The results suggest that higher pump efficiencies, output power, and larger linewidths may be achieved when a mirror with higher effective reflectance is used, and is currently under investigation.

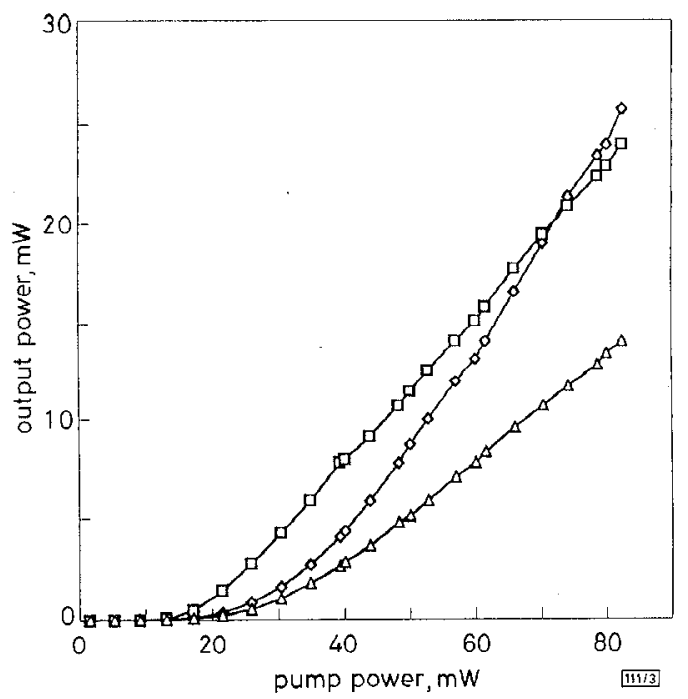

Fig. 3 Measured SFS output power against pump power

\section{$\triangle \mathrm{SPB} 11 \mathrm{~m}$}

$\square$ DPF $6 \mathrm{~m}$

$\diamond$ DPB $11 \mathrm{~m}$

Conclusion: We have experimentally compared efficiencies and the output power of Er-doped superfluorescent fibre sources pumped near $980 \mathrm{~nm}$ in SPB, DPB, and DPF configurations, operating in their optimal mean wavelength regions which are either pumppower independent or pump-power insensitive. It is shown that an SFS in the DPB configuration can have the pump efficiencies $(\sim 52 \%)$, output power $(-26 \mathrm{~mW}$ at $82 \mathrm{~mW}$ pump $)$ and linewidths (27 nm), all greater than those in SPB and DPF configurations. These characteristics potentially enable a stable DPB SFS to be utilised as a desirable light source for navigation-grade FOGs.

\section{(C) IEE 1997}

13 Februcary 1997

Electronics Letters Online No: 19970437

L.A. Wang and C.D. Chen (Institute of Electro-Optical Engineering, National Taiwan University, Taipei, Taiwan, Republic of China)

E-mail: lon@ccms.ntu.edu.tw

\section{References}

1 WYSOCKI, P.F., DIGONNET, M.J.F., KIM, B.Y., and SHAW, H.J.: 'Characteristics of Erbium-doped superfluorescent fibre sources for interferometric sensor applications', J. Lightwave Technol., 1994, 12, (3), pp. 550-567

2 HALL, D.C., and BURNS, W.K.: 'Wavelength stability optimisation in Er-doped superfluorescent fibre sources', Electron. Lett., 1994, 30, (8), pp. 653-654

3 WYSOCKI; P.F., DIGONNET, M.J.F., and KIM, B.Y.: 'Wavelength stability of a highoutput, broadband, Er-doped superfluorescent fibre source pumped near 980nm', Opt. Lett., 1991, 16, (12), pp. 961-963

4 HALL, D.C., BURNS, W.K., and MOELLER, R.P.: 'High-stability Erdoped superfluorescent fibre sources', J. Lightwave Technol., 1995, 13, (7), pp. 1452-1460

5 WANG, L.A., and CHEN, C.D.: 'Stable and broadband Er-doped superfluorescent fibre sources utilising double-pass backward configuration', Electron. Lett., 1996, 32, (19), pp. 1815-1817
6 WANG, L., and CHEN, C.D.: 'Characteristics comparison of Er-doped double pass superfluorescent fibre sources pumped near $980 \mathrm{~nm}$, to be published in Photonics Technol. Lett.

7 Unpublished simulation data.

8 MORKEL, P.R., LAMING, R.I., and PAYNE, D.N.: "Noise characteristics of high-power doped-fibre superluminescent sources', Electron. Lett., 1990, 26, (2), pp. 96-98

\section{Enhanced mechanical performance of singlemode optical fibres irradiated by a CW UV laser}

\author{
D. Varelas, H.G. Limberger and R.P. Salathé
}

\begin{abstract}
Indexing terms: Optical fibres, Mechanical variables measurement
Optical fibres irradiated by a CW UV laser were found to have a mechanical resistance of $5 \mathrm{GPa}$ similar to that of pristine fibre. A comparison of fibre exposed to pulsed and $\mathrm{CW}$ irradiation shows that there is significantly higher mechanical degradation in the pulsed case when comparable doses are used.
\end{abstract}

The effect of mechanical degradation in optical fibres due to pulsed UV laser irradiation has recently been reported $[1,2]$. This phenomenon has important consequences for the reliability of the UV induced Bragg gratings which are used as strain, temperature and pressure sensors in a variety of fields. In this Letter we examine the influence of homogenous irradiation, using a $\mathrm{CW}$ laser in the mechanical degradation of the fibre and we compare it with the equivalent irradiation dose delivered by a pulsed laser source.

The lasers used in our experiment were a $\mathrm{KrF} 248 \mathrm{~nm}$ pulsed excimer laser, and a CW frequency doubled $\mathrm{Ar}^{+} 240 \mathrm{~nm}$ laser. In both cases, the dimensions of the beam which hit the fibre perpendicularly to its axis were evaluated with a beam view analyser. which allowed us to precisely control the doses irradiated. The lateral beam dimension was chosen so that the fibre was irradiated on its entire surface $(200 \mu \mathrm{m})$. To calculate the fluence, the irradiated surface was measured using FWHM intensity values. The mechanical resistance of a $22 \% \mathrm{~mol} \mathrm{GeO}_{2}$-doped optical fibre was investigated. The coating of the fibre was acrylate, which was stripped off before irradiation by exposure to $\mathrm{H}_{2} \mathrm{SO}_{4}$, eliminating any possible influence on the mechanical behaviour of the fibre. Tension tests were carried out, as described in [1], within an interval of $6 \mathrm{~h}$ from the irradiation time, so that humidity and other environmental parameters could not cause mechanical degradation to the fibre: the relative humidity and temperature were similar for both laser exposures. In the $\mathrm{CW}$ case, two different total irradiation doses, 0.5 and $1 \mathrm{~kJ} / \mathrm{cm}^{2}$, were studied. The intensity was $1.3 \mathrm{~W} /$ $\mathrm{cm}^{2}$, and the irradiation times were 385 and $770 \mathrm{~s}$, respectively. In the pulsed case, the fluence per pulse was $150 \mathrm{~mJ} / \mathrm{cm}^{2}$ and the number of irradiated pulses was 3333 and 6666, resulting in total doses of 0.5 and $1 \mathrm{~kJ} / \mathrm{cm}^{2}$, respectively. In both cases the irradiated fibre length was $5 \mathrm{~mm}$ and the breaking stress was measured for a load rate of $45 \mathrm{~kg} / \mathrm{mm}$.

Table 1: Comparative table of mechanical performance of different irradiation parameters

\begin{tabular}{|c|c|c|c|c|c|c|}
\hline & \multirow{2}{*}{$F_{\text {pulse }}$} & \multirow{2}{*}{$F_{\text {iotal }}$} & \multicolumn{3}{|c|}{ Breaking strength } & \multirow{2}{*}{$m$-value } \\
\cline { 4 - 7 } No & $\mathrm{mJ} / \mathrm{cm}^{2}$ & $\mathrm{~kJ} / \mathrm{cm}^{2}$ & $\mathrm{GPa}$ & median & min & \\
irradiation & 0.0 & 0.0 & 5.18 & 5.13 & 4.98 & \multirow{2}{*}{112} \\
\hline \multirow{2}{*}{$\mathrm{CW}$} & & 0.5 & 5.15 & 5.03 & 4.88 & 84.6 \\
& & 1 & 5.08 & 4.96 & 4.79 & 72.8 \\
\hline \multirow{2}{*}{ Pulsed } & \multirow{2}{*}{150} & 0.5 & 2.09 & 1.76 & 1.16 & 7.9 \\
& & 1 & 2.09 & 1.68 & 1.16 & 7.1 \\
\hline
\end{tabular}

Fig. 1 shows Weibull plots comparing the mechanical behaviour of CW and pulsed irradiated fibres: the Weibull distribution of the pristine fibre is given as a reference. The median breaking strengths and the $m$-values resulting from this plot are summarised 